doi.org/10.3114/fuse.2021.08.02

\title{
Three independent evolutionary events of sequestrate Lactifluus species in Australasia
}

\author{
T. Lebel ${ }^{1,2,3^{*}}$, J.A. Cooper ${ }^{3}$, M.A. Castellano ${ }^{4}$, J. Nuytinck ${ }^{5}$ \\ ${ }^{1}$ Botanic Gardens and State Herbarium of South Australia, Hackney Rd, Adelaide, SA 5000, Australia \\ ${ }^{2}$ Royal Botanic Gardens Victoria, Birdwood Avenue, South Yarra, Victoria, 3141 Australia \\ ${ }^{3}$ Mannaki Whenua - Landcare Research, P.O. Box 69040, Lincoln 7640, New Zealand \\ ${ }^{4}$ USDA Forest Service, Northern Research Station, 3200 Jefferson Way, Corvallis, Oregon, USA \\ ${ }^{5}$ Naturalis Biodiversity Center, Darwinweg 2, 2333 CR Leiden, The Netherlands
}

*Corresponding author: Teresa.Lebel@sa.gov.au

\begin{abstract}
Key words: lactarioid new taxa ruffle-like systematics taxonomy

Abstract: Three Australian species with sequestrate basidiome forms are recorded for the first time in the genus Lactifluus based on nuclear ITS-LSU and morphological data. These species represent three rare independent evolutionary events resulting in sequestrate basidiomes arising from agaricoid species in three different sections in two subgenera. All three species have highly reduced basidiome forms, and no species with intermediate forms have been found. Lactifluus dendriticus is unique in the genus in having highly branched, dendritic terminal elements in the pileipellis. We provide full descriptions of two species: Zelleromyces dendriticus (= Lactifluus dendriticus comb. nov.) in Lactifluus subg. Lactifluus sect. Gerardii, and Lactifluus geoprofluens sp. nov. in Lf. subg. Lactifluus sect. Lactifluus. A reduced description is provided for the third, Lactifluus sp. prov. KV181 in Lf. subg. Pseudogymnocarpi sect. Pseudogymnocarpi, as it is currently known from a single sequence.
\end{abstract}

Citation: Lebel T, Cooper JA, Castellano MA, Nuytinck J (2021). Three independent evolutionary events of sequestrate Lactifluus species in Australasi. Fungal Systematics and Evolution 8: 9-25. doi: 10.3114/fuse.2021.08.02

Received: 15 January 2021; Accepted: 15 April 2021; Effectively published online: 2 June 2021

Corresponding editor: P.W. Crous

\section{INTRODUCTION}

Distinguishing species of Lactarius and Lactifluus is difficult enough, but the loss or reduction of macroscopic features in sequestrate species makes them equally problematic to distinguish between genera. In Lactarius, sequestrate basidiome forms are known from three main subgenera: subg. Plinthogalus, subg. Russularia, and subg. Lactarius (Dring \& Pegler 1978, Beaton et al.1984, Desjardin 2003, Nuytinck et al. 2003, Eberhardt \& Verbeken 2004, Verbeken \& Walleyn 2010, Wang et al. 2012, Verbeken et al. 2014a, b, Sang et al.2016, Beenken et al. 2016, De Crop et al. 2017, Vidal et al. 2019). All of the Australasian lactarioid sequestrate forms were historically described in the genera Zelleromyces, Arcangeliella or Gastrolactarius, based on morphology, following Northern Hemisphere circumscriptions of genera. Apart from a regional treatment of species of Zelleromyces by Beaton et al. (1984) for Victoria, in which the descriptions and illustrations to six species were provided, Australian species have been little studied. Bougher \& Syme (1998) provided a further description of Z. daucinus from Western Australia, Grgurinovic (1997) short descriptions and a key to three South Australian species, and Trappe \& Claridge $(2003,2008)$ descriptions of two more species. However, the diversification of sequestrate basidiome forms in Lactarius in Australasia is known to be high, with eight species currently described and a further 20-25 species remaining to be described (Beaton et al.1984, Bougher \& Lebel 2001, Lebel 2002b, Trappe \& Claridge 2003, 2008, Lebel et al. unpub. data). However, no phylogenetic analyses of subgenus or sectional placement has as yet been completed for these taxa or the related epigeal taxa, and species circumscription remains based on morphology.

While conducting research on Australasian sequestrate lactarioid taxa and the Lactifluus clarkeae species complex, DNA sequencing revealed species with affinities to Lactifluus rather than Lactarius. This unexpected finding led us to study their morphological characters in detail, test species delimitation and further examine the phylogenetic relationships between agaricoid and sequestrate Lactifluus species, to determine whether sequestrate forms in Lactifluus are the result of independent evolutionary events, similar to the pattern seen in Lactarius (Verbeken et al. 2014a, Sheedy et al. 2016). Here we provide descriptions where possible and confirm placement of the taxa in different subgenera within Lactifluus.

\section{MATERIALS AND METHODS}

\section{Morphology}

Several hundred collections from Australasian herbaria and fungaria (MEL, PERTH, BRI, AD, HO, CANB, PDD) and material held at Oregon State Herbarium (OSC) were examined from diverse geographic regions and habitat types across Australia. Detailed coordinate and elevation data for collections mentioned here are available online through the Atlas of Living Australia 
(https://www.ala.org.au/). Collections matching morphological characters of the known Lactifluus related taxa, or with typical Lactifluus pileipellis structure and cystidia form (approximately 35 collections), were examined in greater detail and sampled for DNA sequencing. Macroscopic characters are described and measured from fresh material, field notes of other collectors, or dried herbarium collections. Measurements taken using dried fungarium material are listed as such and are estimated to be approximately $30 \%$ smaller than measurements taken from fresh specimens. This estimate is based on fresh and dry weights of 30 recent collections of lactarioid sequestrate basidiomes. Colours are described in general terms from field observations in daylight conditions. Habitat, associated plant communities, sporulating season, presence and nature of latex, fresh odour, and taste are based on field notes.

Microscopic characters are described from examination of dried fungarium material. Hand-cut sections were rehydrated in $5 \% \mathrm{KOH}$ solution then mounted in congo red to observe the hymenium, trama, and pileipellis. Spore size, shape, ornamentation and amyloidity were observed in lamellae tissue mounted in Melzer's reagent. Measurements of microscopic characters were taken on an Olympus BX-52 microscope at $\times 400$ or $\times 1000$ using an Olympus DP-73 camera attachment and measurement tools in cellSens standard (v. 1.16). Microscopic measurements are given as a raw range of length $\times$ width with mean \pm standard deviation (SD) of $n$ measurements in parentheses. The length/width quotient (Q) of individual spores is presented as the raw range of $Q$ values with mean \pm standard deviation (SD) of $n$ measurements in parentheses. Basidia, basidioles, and cystidia measurements are given as length (not including sterigmata) $\times$ width at widest point, and width at base or apex. Pseudocystidia, laticiferous hyphae, and hyaline hyphae measurements are given as a raw range of diameters. In highly reduced sequestrate basidiomes there is some difficulty in determining position of cystidial elements, thus in descriptions provided here they are referred to as hymenial cystidia.

Scanning electron microscopy (SEM) of gold-sputtered basidiospores mounted on carbon tape was performed using a Thermo Fisher Scientific XL30 FEG microscope (Waltham, USA) at the University of Melbourne Biosciences Microscopy Unit.

All photographs are based on the type collections unless otherwise stated. Names of fungaria are abbreviated according to Thiers (2011) (http://sweetgum.nybg.org/ih/ - continuously updated). We use the abbreviation ' $L f$.' to distinguish Lactifluus from Lactarius ('L.') when discussing species in these genera.

\section{Molecular studies}

Protocols for DNA extraction (Qiagen Plant DNeasy kit, Germantown, USA or the EZNA forensic kit, Omega Biotek, Norcross USA, for samples older than 1995), PCR, and sequencing followed those in Lebel \& Syme (2012) and Lebel et al. (2015) and the references therein. Assembly, manual editing, and preliminary alignment of sequences were performed within Geneious v. 9.1.7 (Biomatters Ltd). Individual alignments for the ITS and LSU were then manually trimmed in BioEdit v. 7.1.3 (Hall 2011) and some final manual editing done in Geneious v. 9.1.7. The concatenated alignment and phylogenetic trees are available from the Landcare Research datastore https://doi. org/10.7931/n4fc-4z93.

Sequences of the ITS and LSU from similar species (based on blast searches), and representative taxa to cover subgenera and sections within Lactifluus were retrieved from GenBank and UNITE (Kõljalg et al. 2013), to generate a concatenated alignment. This was done with the on-line version of MAFFT v. 7 (Katoh et al. 2019). Three species of Multifurca were utilised as outgroup. Novel sequences representing collections from Australasia and other regions generated for this study are listed in Table 1 with relevant GenBank accession numbers, and all sequences utilised in analyses. Sequences labelled as provisional taxa " $L f$. sp. 1-13" are numbered according to a broader analysis of Australasian species of Lactifluus which will be published elsewhere; we have retained the numbering convention for consistency.

Phylogenetic analyses of the concatenated ITS+LSU alignment was performed with Maximum Likelihood (ML) in RAxML v. 8.2.12 (Stamatakis et al. 2014) using the CIPRES Science Gateway v. 3.3 (Miller et al. 2010). The final alignment comprised 211 specimens (195 ITS and 146 LSU sequences), consisting of $2087 \mathrm{bp}$ including gaps. Gaps in alignments were treated as missing data. The tree was visualised in FigTree v. 1.4.2 (Rambaut 2009).

\section{RESULTS}

Sequestrate forms arose independently in at least three sections in two subgenera of Lactifluus: subg. Lactifluus sect. Gerardii (Lf. dendriticus comb. nov.) and sect. Lactifluus (Lf. geoprofluens sp. nov.), and subg. Pseudogymnocarpi sect. Pseudogymnocarpi (Lf. sp. prov. KV181). Lactifluus dendriticus comb. nov. appears to be a wide-spread species from eastern Australia, ectomycorrhizal with Eucalyptus and Acacia. It is in a strongly supported clade, sister to $L f$. wirrabara, three provisional Australian species with agaricoid basidiomes (Lf. sp. prov. 10-12), an environmental sequence with unknown basidiome form $L f$. sp. prov. 13 from Australia, $L f$. limbatus from Malaysia, $L f$. sinensis from China, $L f$. coniculus from Sri Lanka, and $L f$. midnapurensis from India in section Gerardii (Fig. 1). All of these Asian taxa are associated with lowland tropical rainforest dominated by Dipterocarp taxa (Stubbe et al. 2012, Song et al. 2017, Phookamsak et al. 2019).

Lactifluus geoprofluens sp. nov. is in a weakly supported clade with two agaricoid species $L f$. jetiae nom. prov. (Fig. $3 C$ ) and $L f$. rugulostipitatus nom. prov. (in press; these provisional species will be published elsewhere), a third provisional species known only from environmental sequences ( $L f . s p$. prov. 8), several undescribed taxa with agaricoid basidiomes from Thailand and Japan, and $L f$. distantifolius and Lf. Longipilus (Fig. 2). More data are required to reliably recognise sister relationships as branch support values are low in this mixed clade of mostly undescribed taxa.

A single sequence of the sequestrate Lactifluus sp. prov. KV181 is in a clade with a mix of species from diverse geographic locations, $L f$. pseudoluteopus from Thailand, Lf. hygrophoroides from North America, Lf. rugatus from Southern Europe, and $L f$. holophyllus and $L f$. luteolamellatus from South Korea in sect. Pseudogymnocarpi. While we did take a small sample for DNA in the field, unfortunately, the single collection of $L f$. sp. prov. KV181 was destroyed by mould contamination after drying and discarded, and no further collections of this sequestrate taxon were found while looking through abundant material of sequestrate lactarioid taxa in herbaria. Thus while a full description cannot be provided at this time, we do provide a photo of basidiomes, and a partial description, in the hope that further material will be found. 
Table 1. Specimens used in the phylogenetic analysis, including infrageneric taxon, species (as originally identified in the field or as labelled in GenBank), herbarium numbers and typification, country of origin, and ITS/LSU GenBank accession numbers. New sequences generated for this study are indicated in bold. Abbreviations used: AU - Australia, NZ - New Zealand, NCal - New Caledonia, WA - Western Australia, VIC - Victoria, TAS Tasmania, SA - South Australia, NT - Northern Territory, NSW - New South Wales, QLD - Queensland, Frlsland - Fraser Island.

(Infrageneric) taxon Species Herbarium or collection number Country

GenBank accession numbers

\section{ITS}

LSU
Multifurca

Multifurca ochricompacta

Multifurca sp.

Multifurca stenophylla

Multifurca zonaria

subg. Gymnocarpi sect. Lactifluus foetens

Gymnocarpi

Lactifluus gymnocarpus

Lactifluus tanzanicus

subg. Gymnocarpi sect. Luteoli

Lactifluus brunneoviolascens

Lactifluus luteolus

Lactifluus russulisporus

subg. Gymnocarpi sect. Nebulosi

Lactifluus chiapanensis

Lactifluus guadeloupensis

subg. Gymnocarpi sect. Lactifluus panuoides

Panuoidei

subg. Gymnocarpi sect. Phlebonemi

subg. Gymnocarpi sect. Tomentosi

Lactifluus aff. phlebonemus

Lactifluus albens
Lactifluus aurantioruber

Lactifluus clarkeae

Lactifluus clarkeae

Lactifluus flocktonae

Lactifluus psammophilus

Lactifluus pseudoflocktonae

Lactifluus sp. 1

Lactifluus sp. 5

Lactifluus sp. 6

subg. Lactariopsis sect. Albati

subg. Lactariopsis sect. Edules Lactariopsis

subg. Lactariopsis sect. Neotropicus

subg. Lactifluus sect. Allardii

subg. Lactifluus sect. Ambicystidiati

subg. Lactifluus sect. Gerardii

Lactifluus velutissimus

Lactifluus venezuelanus

Lactifluus allardii

Lactifluus ambicystidiatus

Lactifluus auriculiformis subg. Lactariopsis sect.
Lactifluus caliendrifer

BB02107

MEL238568

$\mathrm{n} / \mathrm{a}$

CWD584

FH12-009

ADK3688 BR

EDC12-047 GENT

TS1277

AV13-038 GENT

KW378 GENT Holotype

AV05-253 GENT

REH9398 NY Holotype

V.M.Bandala 4374A GENT

RC_Guad11-023 LIP Holotype

G128

EDC12-023 GENT

MEL2231695 Type

MEL2257827

MEL2101947 Epitype

PDD102596

MEL2238290 Epitype

MEL2238407 Type

MEL2238269 Holotype

environmental sample

CMMy30M1

PGK13-130 Nothofagus

environmental sample KT-26

Tristaniopsis

UE20.09.2004-22 UPS

FN05-628 GENT

BB00-1518 PC

JD886

RC_Gaud11-017 LIP

AV05-286 GENT

AU

AU

AU

NZ

$n / a$

KUN_F88179

AV12-050 GENT Holotype
Thailand

Benin

Cameroon

Tanzania

Italy

Thailand

USA

Mexico

Guadeloupe

Guyana

Cameroon

KR364062

KR364191

AU_WA

MW134740

MW128112

AU_TAS

AU_SA

AU_VIC

AU_VIC

AU_VIC

New Caledonia

MW134745

MW128115

MW134754

MW128120

MW134770

MW128130

JX266621

JX266637

MW134791

MW128144

MW134801

MW128151

KY774240

$\mathrm{n} / \mathrm{a}$

New Caledonia

KP691436

KR605507

New Caledonia

LC271308

n/a

DQ422034

DQ422034

Malawi

KR364020

KR364147

Madagascar

AY606981

KR364253

Congo

KR364075

KR364204

Guadeloupe

KP691411

KP691420

USA

KF220015

KF220124

China

KR908670

KR908672

Thailand

KR364086

KR364216 
Table 1. (Continued).

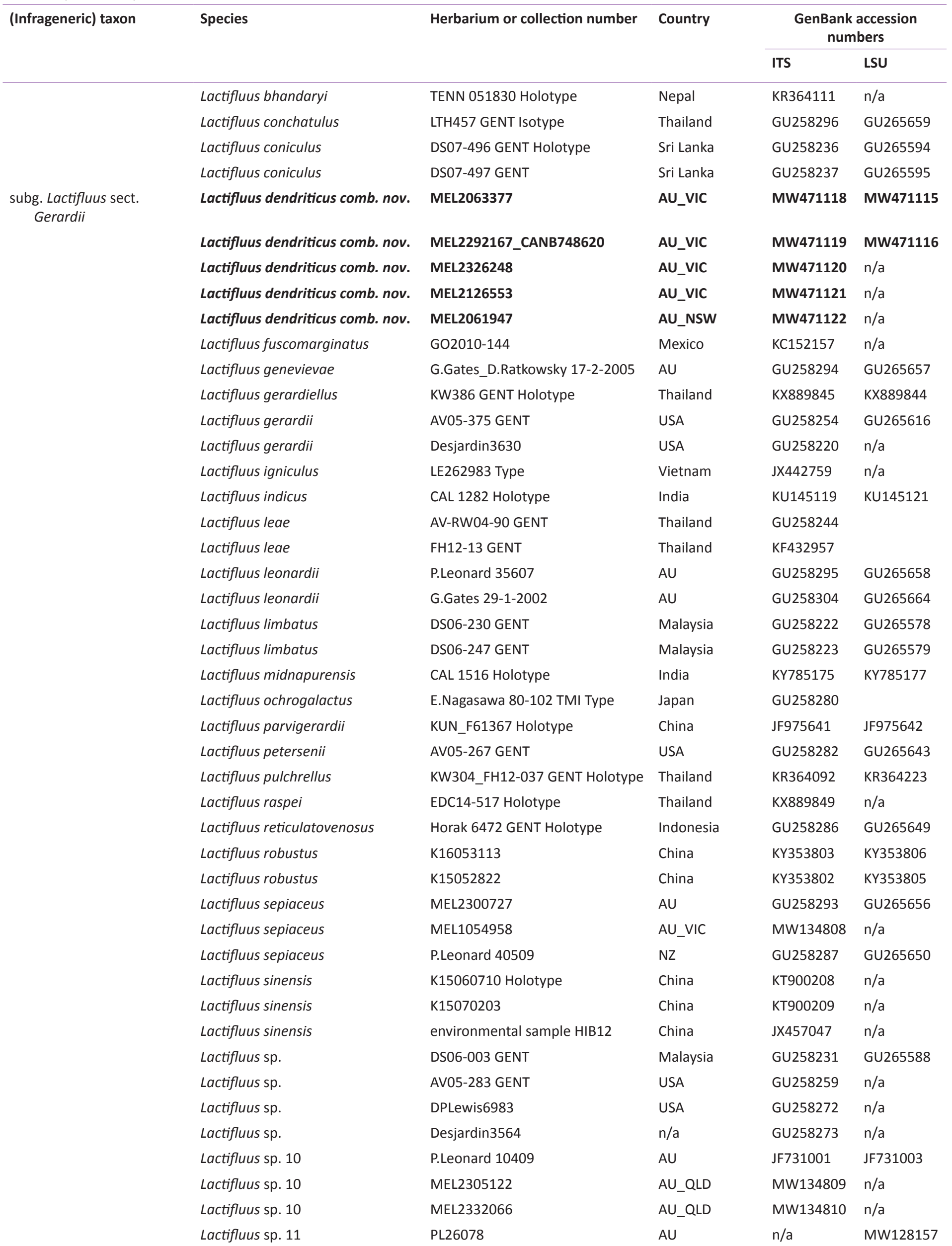


Table 1. (Continued).

\begin{tabular}{|c|c|c|c|c|c|}
\hline \multirow[t]{2}{*}{ (Infrageneric) taxon } & \multirow[t]{2}{*}{ Species } & \multirow[t]{2}{*}{ Herbarium or collection number } & \multirow[t]{2}{*}{ Country } & \multicolumn{2}{|c|}{$\begin{array}{c}\text { GenBank accession } \\
\text { numbers }\end{array}$} \\
\hline & & & & ITS & LSU \\
\hline & Lactifluus sp. 13 & environmental sample RFLP61 & $\mathrm{AU}$ & DQ388868 & $\mathrm{n} / \mathrm{a}$ \\
\hline & Lactifluus sp. 13 & environmental sample Toosoil16 & $\mathrm{AU}$ & KC222796 & $\mathrm{n} / \mathrm{a}$ \\
\hline & Lactifluus subgerardii & AV05-389 GENT & USA & GU258271 & $\mathrm{n} / \mathrm{a}$ \\
\hline & Lactifluus wirrabara & G.Gates_D.Ratkowsky 12-07-2003 & $\mathrm{AU}$ & GU258306 & GU265666 \\
\hline & Lactifluus wirrabara & G.Gates_D.Ratkowsky 17-01-2002 & $\mathrm{AU}$ & GU258305 & GU265665 \\
\hline & Lactifluus wirrabara & G.Gates_D.Ratkowsky 24-01-2004 & $\mathrm{AU}$ & GU258307 & $\mathrm{n} / \mathrm{a}$ \\
\hline & Lactifluus wirrabara & JET943 MEL & $\mathrm{AU}$ & GU258291 & $\mathrm{n} / \mathrm{a}$ \\
\hline \multirow{27}{*}{$\begin{array}{l}\text { subg. Lactifluus sect. } \\
\text { Lactifluus }\end{array}$} & Lactifluus corrugis & JN2004-015 GENT & USA & JQ753820 & JQ348262 \\
\hline & Lactifluus corrugis & AV05-291 GENT & USA & JQ753823 & JQ348266 \\
\hline & Lactifluus crocatus & LTH268 GENT & Thailand & HQ318266 & HQ318181 \\
\hline & Lactifluus crocatus & LTH202 GENT & Thailand & HQ318248 & HQ318157 \\
\hline & Lactifluus dissitus & AV-KD-KVP09-082 GENT & India & $\mathrm{n} / \mathrm{a}$ & JN389035 \\
\hline & Lactifluus distantifolius & DS07-461 GENT Isotype & Thailand & HQ318223 & HQ318124 \\
\hline & Lactifluus distantifolius & LTH288 GENT & Thailand & HQ318274 & HQ318193 \\
\hline & Lactifluus geoprofluens sp. nov. & MEL2145804 Holotype & AU_VIC & MW471123 & MW471117 \\
\hline & Lactifluus jetiae & MEL2238281 Holotype & AU_VIC & MW134811 & MW128158 \\
\hline & Lactifluus mexicanus & Montoya5276 Holotype & Mexico & MK211181 & MK211190 \\
\hline & Lactifluus oedematopus & AV07-079 GENT & Belgium & JQ753835 & JQ348270 \\
\hline & Lactifluus oedematopus & RW1228 GENT & France & HQ318216 & HQ318116 \\
\hline & Lactifluus oedematopus & KVP12-001 GENT Neotype & Germany & KR364100 & KR364232 \\
\hline & Lactifluus pagodicystidiatus & MEL2121979 & $A U \_V I C$ & MW134815 & MW128161 \\
\hline & Lactifluus pagodicystidiatus & MEL2150777 Holotype & AU_VIC & MW134816 & MW128162 \\
\hline & Lactifluus pallidilamellatus & Leticia Montoya 4716 & Mexico & JQ753824 & JQ348268 \\
\hline & Lactifluus pinguis & LTH117 GENT Holotype & Thailand & HQ318211 & HQ318111 \\
\hline & Lactifluus pinguis & LTH169 GENT & Thailand & HQ318221 & HQ318121 \\
\hline & Lactifluus rugulostipitatus & MEL2329677 Holotype & AU_NT & MW134817 & MW128163 \\
\hline & Lactifluus rugulostipitatus & MEL2329673 & AU_NT & MW134819 & $\mathrm{n} / \mathrm{a}$ \\
\hline & Lactifluus sp. & KIINA158 GENT & China & HQ318225 & HQ318126 \\
\hline & Lactifluus sp. & AV-KD-KVP09-134 GENT & India & JN388978 & JN389026 \\
\hline & Lactifluus sp. & AV-KD-KVP09-128 & India & $\mathrm{n} / \mathrm{a}$ & JN389020 \\
\hline & Lactifluus sp. & AV-KD-KVP09-137 & India & $\mathrm{n} / \mathrm{a}$ & JN389027 \\
\hline & Lactifluus sp. & AV-KD-KVP09-129 & India & $\mathrm{n} / \mathrm{a}$ & JN389021 \\
\hline & Lactifluus sp. & OSA-My-3993 & Japan & $\mathrm{n} / \mathrm{a}$ & AB238645 \\
\hline & Lactifluus sp. & OSA-My-3998 & Japan & $\mathrm{n} / \mathrm{a}$ & AB238650 \\
\hline
\end{tabular}


Table 1. (Continued).

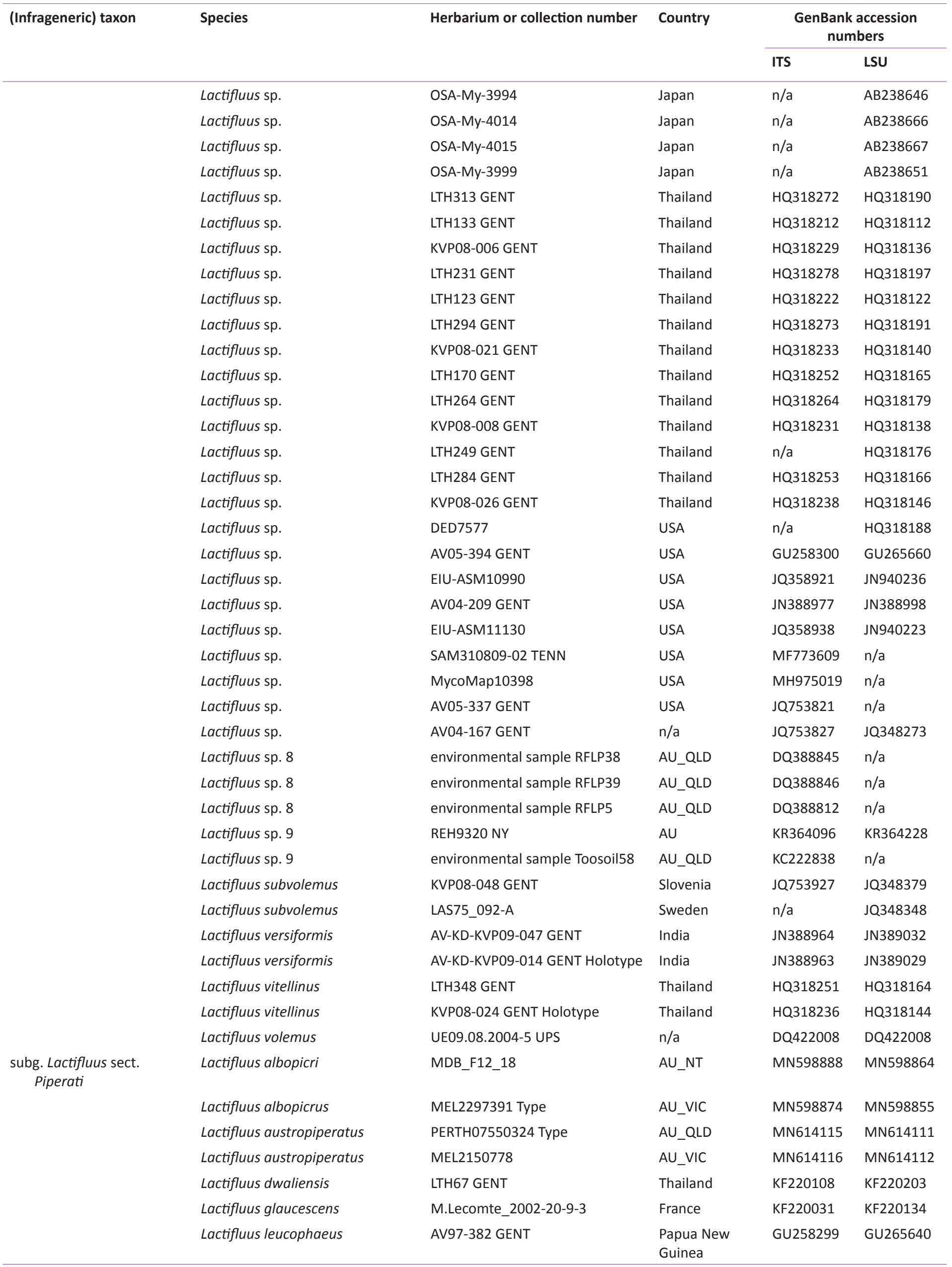


Table 1. (Continued).

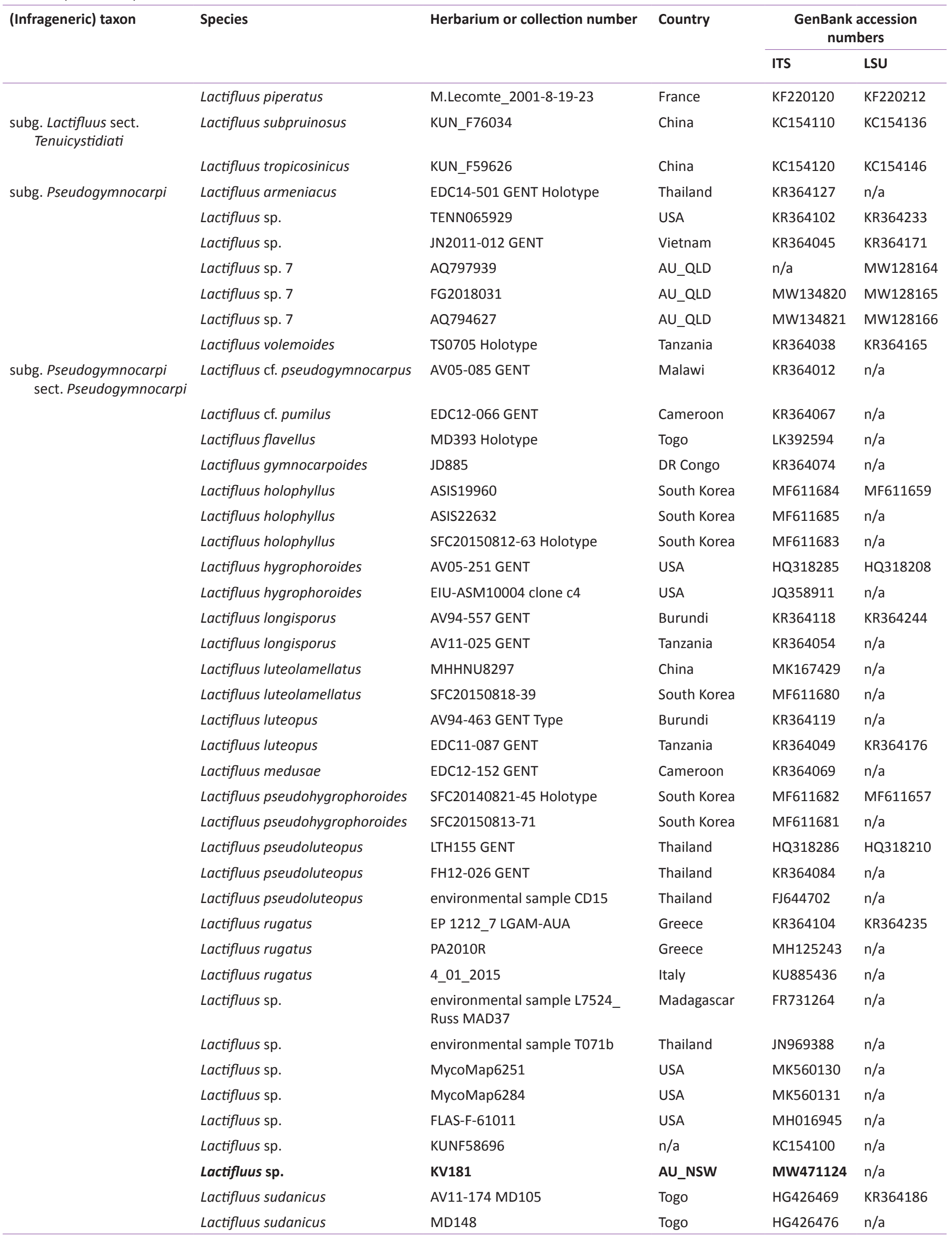




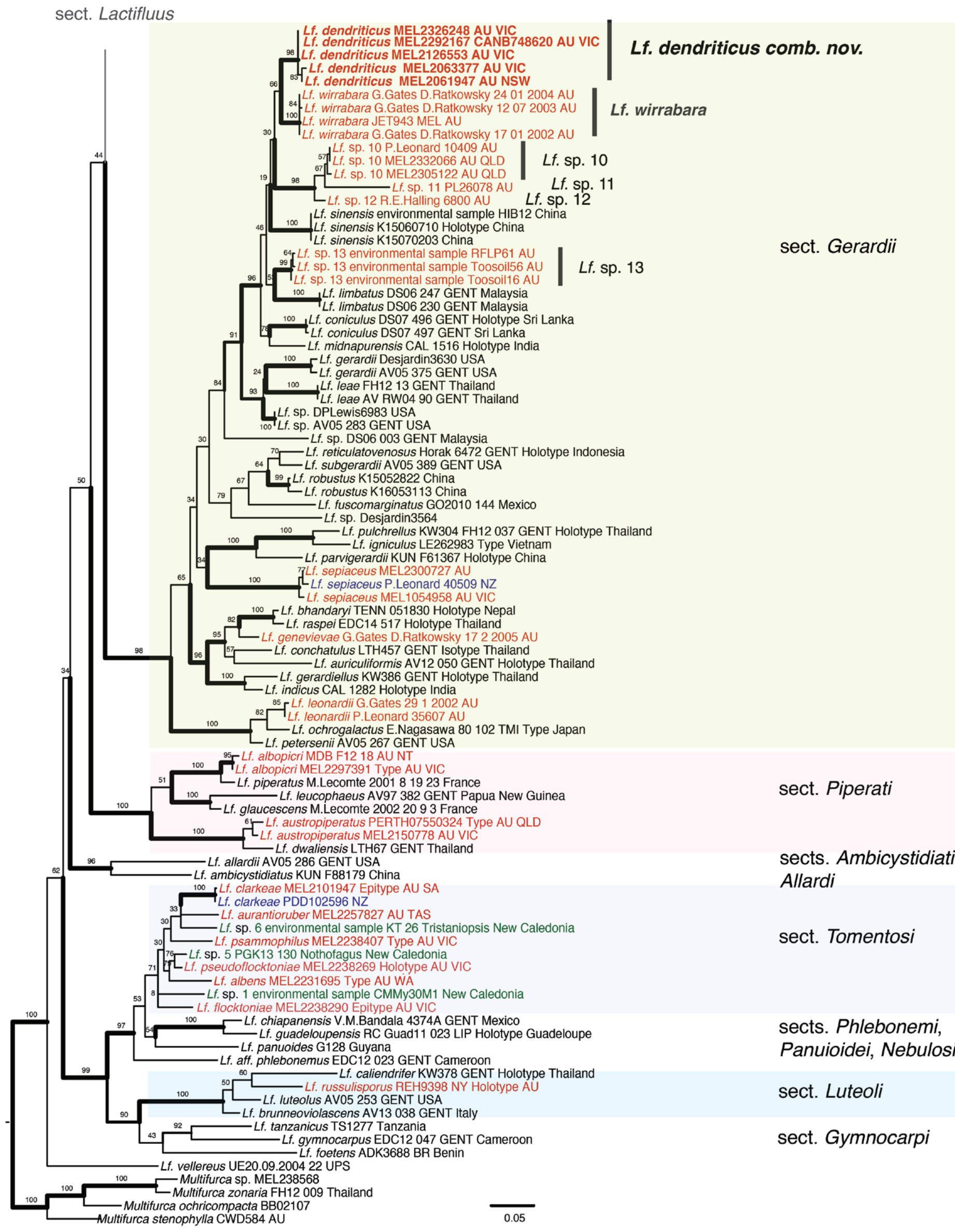

Fig. 1. Maximum Likelihood tree based on ITS and LSU sequences for subgenus Lactifluus sections Gerardii, Piperati, Ambicystidiati, and Allardi; subgenus Gymnocarpi sections Tomentosi, Phlebonemi, Panuioidei, Nebulosi, Luteoli, Gymnocarpi, and Lf. vellereus from Lf. sect. Albati, subg. Lactariopsis, with outgroup Multifurca. Bold lines indicate ML support $\geq 90 \%$. Bold text sequences of sequestrate taxa generated for this study. Red text: Australian specimens or sequences, blue text: New Zealand specimens, green text: New Caledonia specimens or sequences. 


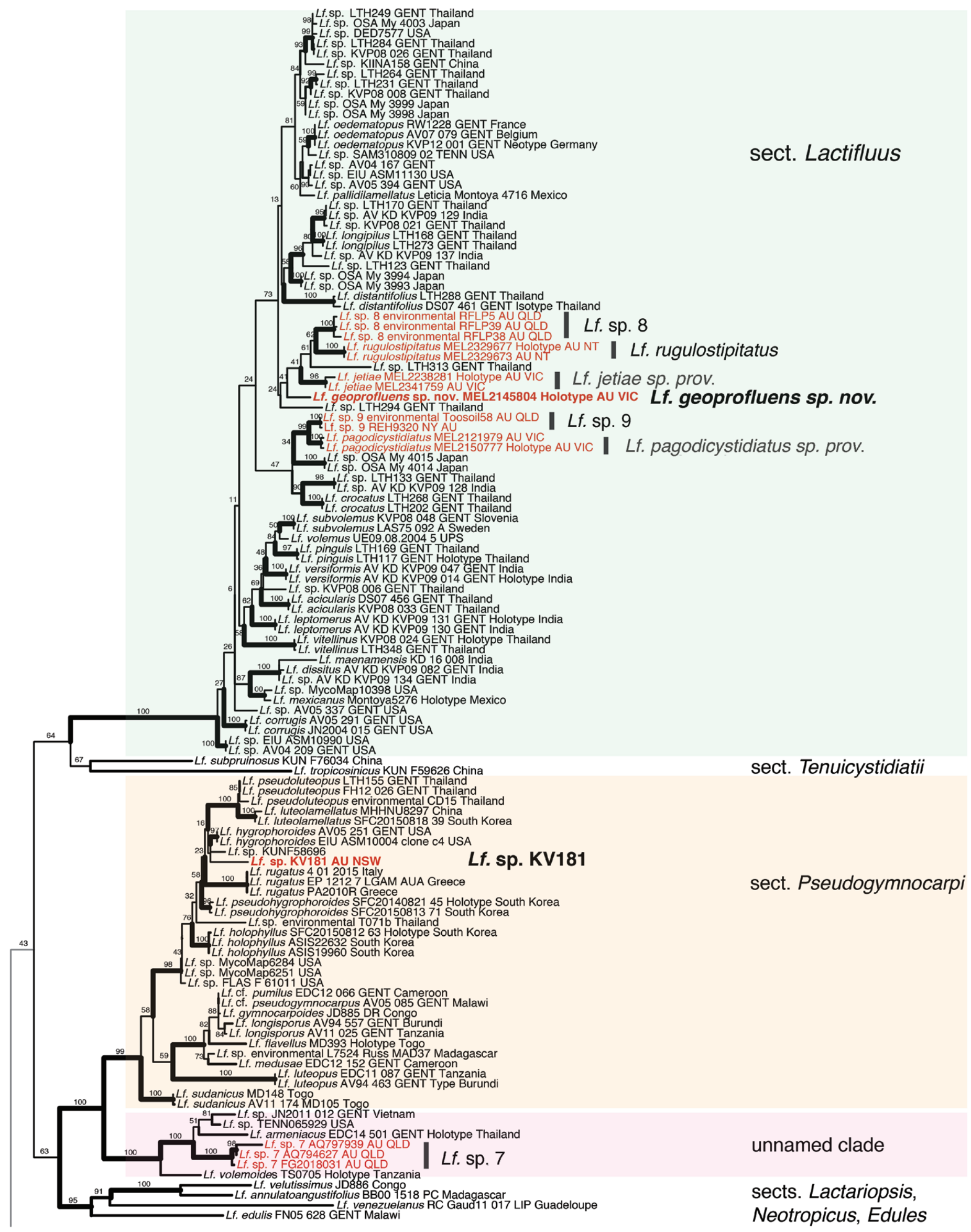

sect. Gerardii

0.05

Fig. 2. Maximum Likelihood tree based on ITS and LSU sequences for subgenus Lactifluus sections Lactifluus and Tenuicystidiati; and subgenus Pseudogymnocarpi section Pseudogymnocarpi and an unnamed clade, and exemplars from sections Lactariopsis, Neotropicus and Edules. Bold lines $\mathrm{ML} \geq 90 \%$. Bold text sequences generated for this study. Red text: Australian specimens or sequences. 


\section{Taxonomy}

\section{Section Gerardii}

Lactifluus dendriticus (T. Lebel) T. Lebel, J. Cooper \& Nuytinck, comb. nov. MycoBank MB 838127. Figs 3B, 4A-F.

Basionym: Zelleromyces dendriticus T. Lebel, Australasian Mycologist 21: 4. 2002. figs 1-4. MB 373383.
Etymology: The specific epithet, "dendriticus" (L), refers to the dendritic terminal element of the pileipellis.

Diagnosis: This species can be distinguished by the pale sequestrate basidiomes, unique dendritic terminal elements forming a short turf in the pileipellis, and globose spores.

Typus: Australia, Victoria, Errinundra National Park, The Gap Scenic Reserve, Gunmark Road, $1.3 \mathrm{~km}$ southeast of Survey
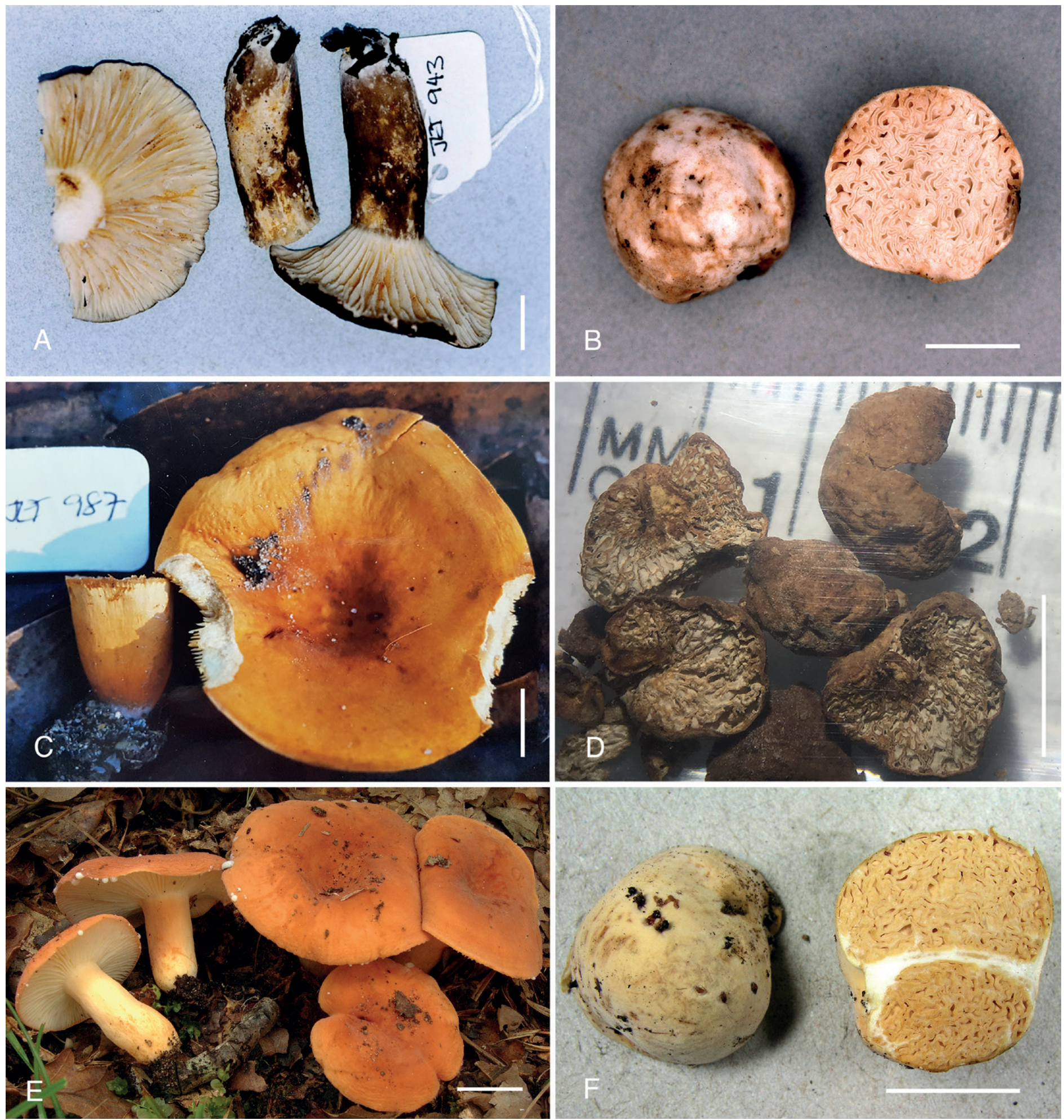

Fig. 3. Basidiomata of new sequestrate species and representative agaricoid species. A. Lactifluus wirrabara (JET 943; photo J.E. Tonkin). B. Lf. dendriticus (photo T. Lebel). C. Lf. jetiae (photo J.E. Tonkin). D. Lf. geoprofluens (photo L. Vaughan). E. Lf. rugatus (photo U. Pero). F. Lf. sp. prov. KV181 (photo T. Lebel). Scale bars $=10 \mathrm{~mm}$ 

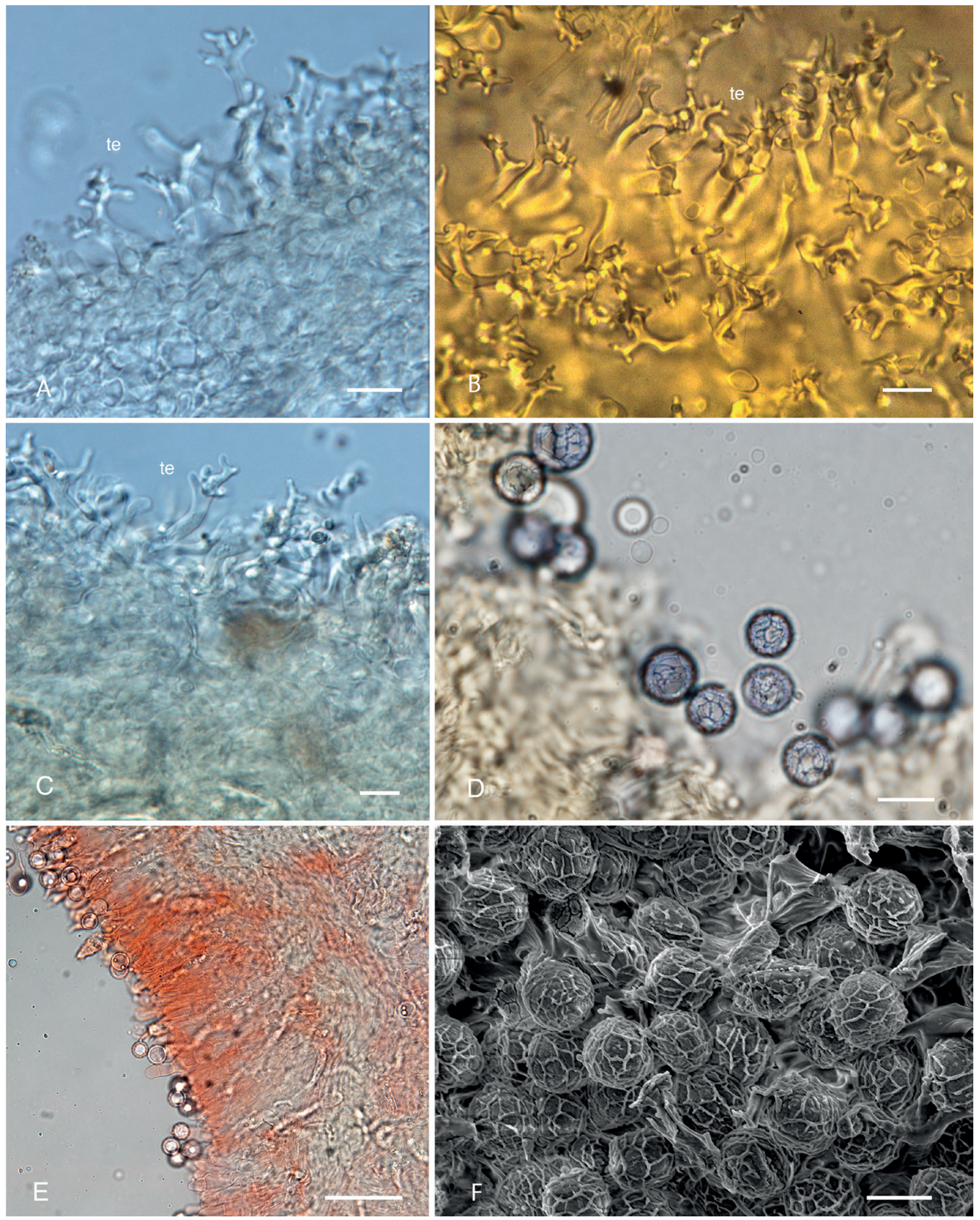

Fig. 4. Lactifluus dendriticus. A. Cross-section of pileipellis with dendritic terminal elements. B. Scalp section showing dendritic terminal elements. C. Dendritic terminal elements. D. Basidiospores. E. Hymenial trama with pseudocystidia. F. Scanning Electron Microscopy photo micrograph of basidiospores. Scale bars: A-D, $F=10 \mu \mathrm{m}, \mathrm{E}=50 \mu \mathrm{m}$. 
Road, Claridge site 124, 28 May 1996, A. Jumpponen \& J. Trappe 18478 (holotype MEL 2063482, isotype OSC 80519).

Basidiomes hypogeal, sequestrate, 5-25 × 5-20 mm, globose to subglobose, slightly irregular or furrowed near point of attachment; locules exposed at point of attachment in some basidiomata as pileus is incomplete, surface dry, smooth to minutely fibrillose or verrucose, white with a faint yellowish tinge and often with small darker yellow-brown patches, pileipellis thin, off-white in cross-section. Hymenophore loculate, locules large $0.5-2 \mathrm{~mm}$ diam, somewhat irregular, white when young, becoming pale yellow to pale brown-yellow with maturity, pale tan when dried. Stipe-columella absent. Latex scant to abundant, white, unchanging. Taste mild. Odour mildly sweet, pleasant. Basidiospores 9.0-10.8 $\times$ 8.5-10.6 $\mu \mathrm{m}(\bar{x}=9.8 \pm 0.53$ $\times 9.69 \pm 0.64, \mathrm{n}=50)$, globose $[\mathrm{Q}=1.0-1.05(\bar{x}=1.01 \pm 0.02, n$ $=50)]$, walls weakly amyloid, ornamentation a fine, complete to almost complete regular mesh reticulum $\pm 0.5-0.7 \mu \mathrm{m}$ high, with few scattered, isolated warts also present; hilar appendix small, central, plage absent. Basidia 33-65 × 7-13 $\mu \mathrm{m}$, elongated clavate to cylindrical or rarely narrowly ventricose, hyaline, thinwalled, with 2 or 4 robust, slightly curved sterigmata 4-10 $\times 2-3.5$ $\mu \mathrm{m}$. Hymenophoral trama 14-22 $\mu \mathrm{m}$ wide, a narrow central strand of crowded, hyaline hyphae 2-4 $\mu \mathrm{m}$ diam, with slightly thickened walls intermixed with scattered sinuous laticiferous hyphae 3-7.5 $\mu \mathrm{m}$ diam, sphaerocytes absent; subhymenium 15-35 $\mu \mathrm{m}$ wide, with two or three tiers of \pm isodiametric cells 8-15 $\mathrm{mm}$ diam. Pseudocystidia absent or when present, 5-11 $\mu \mathrm{m}$ diam, thin-walled, cylindrical to narrowly clavate, scattered, with oily refractive contents; arising in trama from laticiferous hyphae extending up through the subhymenium. Pileipellis 18$45 \mu \mathrm{m}$ wide, composed of a very narrow cutis with an almost complete turf of thick-walled (throughout length) dendritic terminal elements and scattered rare laticiferous hyphae when young, this turf becoming patchy as basidiomata expands; no gelatinous matter or very little in some basidiomes apparent amongst terminal elements dendritic terminal elements less branched and intricate when young, variously and often elaborately branched when older, coralloid to irregularly repeatedly branched with coralloid tips, 18-32 $\mu \mathrm{m}$ high, 4-25 $\mu \mathrm{m}$ wide, 3-6 $\mu \mathrm{m}$ at base, walls thick. Pileus trama 32-60 $\mu \mathrm{m}$ wide, of tightly interwoven, subgelatinous, hyaline hyphae 2-4 $\mu \mathrm{m}$ diam, more crowded towards the surface than within, with scattered to common, sinuous laticiferous hyphae 3-8 $\mu \mathrm{m}$ diam, refractive in $\mathrm{KOH}$; sphaerocytes absent.

Ecology and Distribution: Sporulating in April-July in small to large groups, at mid to higher elevations in the mountains of south eastern Australia in Victoria, Tasmania, and New South Wales. Hypogeal in mixed forests of Eucalyptus fastigata, E. cypellocarpa, E. radiata, E. dalrympleana, E. globoidea, E. obliqua, E. pauciflora, E. stellulata, E. regnans, Acacia dealbata, A. melanoxylon, A. aculeatissima, A. cognata, or A. mearnsii.

Additional specimens examined: Australia, Victoria, Kinglake National Park, 120 m down Mountain Creek Track, 8 Jul. 1993, M.A. Castellano OSC 80520 (MEL 2063479); East Gippsland, Errinundra National Park, The Gap Scenic Reserve, Gunmark Road, $1.3 \mathrm{~km}$ SE of Survey Road, Claridge Site 124, 30 May 2003, A.W. Claridge, W. Colgan III, A. Jumpponen, I. Kratzer AWC5079 (CANB 748631); ibid., Gap Road, $50 \mathrm{~m}$ E of Bonang River Bridge, Claridge Site 117, 9 May 2003, A.W. Claridge AWC4731 (CANB 65451); ibid. Claridge Site 117, 27 May 1999, J.M. Trappe AWC2589 (MEL 2105044); ibid., 27
May 1999, J.M. Trappe AWC2588 (MEL 2105043); ibid., Gap Road, 3.9 km E of Bonang Highway, Claridge site 118, 28 May 1996, J.M. Trappe 18434 (MEL 2063477); ibid., Gap Road, 2.1 km west of track to Result Creek Falls, Claridge Site 138, 11 Jun. 1996, J.M. Trappe 19022 (MEL 2063472); ibid., Gap Road, $5.2 \mathrm{~km}$ E of Junction with Bonang Highway, Claridge Site 119, 9 May 2003, J.M. Trappe \& A.W. Claridge AWC4741 (DAR 76661, CANB 748620, MEL 2292167) (also in K, NY, BPI, FH); ibid., Claridge Site 119, 26 May 2001, T. Lebel AWC3994 (CANB 736252); ibid., Gap Road, 2.1 km west of track to Result Creek Falls, Claridge Site 138, 11 Jun. 1996, A.M. Jumpponen JMT19021 (MEL 2063473); Rich Forest Management Block, Jack Road, $0.7 \mathrm{~km}$ N of Winter Road, Claridge Retrospective Study Site R19, 27 Apr. 1996, A.W. Claridge AWC352 (CANB 669623); Lind National Park, Euchre Valley Road, $0.2 \mathrm{~km}$ W of Junction with Lind Park Road, Claridge Site 74, 29 May 1999, A.W. Claridge AWC2746B (MEL 210507); Murrungowar Forest Management Block, Princes Highway, $0.4 \mathrm{~km}$ E of Junction with Bendoc Ridge Road, Claridge Site 64, 26 May 1999, T. Lebel AWC2556 (MEL 2105041); 2.4 km N of Baw Baw National Park,16 Jun. 1994, J.M. Trappe H6792 (PERTH 7593031); 20 km west of Mansfield near Mt Buller, 9 Jul. 1993, T. Lebel JMT14063 (MEL 2063478); Alpine National Park, Black Mountain Road, Rams Horn Track. Claridge Site No. 78, 11 Nov. 2008, A.W. Claridge JMT18089 (MEL 2326248); Noojee State Park, ca. $1 \mathrm{~km}$ from Tooronga Road, along Link Road in small carpark area, 25 Feb. 2002, S.H. Lewis 791 (MEL 2061947); North east of Marysville on Lady Talbot Drive, wishing Well Track, 19 Apr. 1999, T. Lebel TL5 (MEL 2063377); New South Wales, Bombala, Bondi Gulf Nature Reserve, Bondi Gulf Road, 4 km E of Cann Valley Highway, Claridge Site 53, 15 Jun. 1999, J.M. Trappe AWC2972 (CANB 748185); Tasmania, Break O'Day, Elephant Pass, 16 kms southeast of St Marys, 2 May 1990, N. Malajczuk MEL 2063481; Elephant Pass, 2 May 1990, J.M. Trappe H1373 (PERTH 7673167).

Notes: A revised description of Lactifluus dendriticus is provided here to enable better comparisons to other taxa, and to incorporate more data from fresh collections. Detailed illustrations of spores, pileipellis and hymenophore structure and constituent elements were provided as part of the original description (Lebel 2002). Lactifluus dendriticus is widespread in eucalypt forests occurring in regions with relatively higher rainfall. Its basidiomata do not always produce a latex when cut or bruised. Microscopically, Lactifluus dendriticus is unique, with distinct highly branched pileal terminal elements (Fig. 4A-C) that have not been observed in any other species of sequestrate or currently known agaricoid Russulales taxa.

Taxa in the larger clade, Lactifluus wirrabara, Lf. limbatus, Lf. coniculus, $L f$. sinensis, and $L f$. midnapurensis all have brownish agaricoid basidiocarps (Fig. 3A), and spores with a low almost complete to complete reticulum; however these species have smaller, subglobose to broadly ellipsoid spores, and very different pileicystidia or pileal terminal elements.

\section{Section Lactifluus}

Lactifluus geoprofluens T. Lebel, Castellano, Claridge \& Trappe, sp. nov. MycoBank MB 838126. Figs 3D, 5A-F.

Etymology: The species epithet, "profluens", from Latin, meaning "freely flowing" referring to the abundantly flowing latex in cut basidiomes of this species, and "geo" in reference to the hypogeal basidiomes.

Diagnosis: Differs from other currently known lactarioid sequestrate taxa by the faint fishy odour, abundant white latex, globose to occasionally barely subglobose basidiospores, up to 
$10.5 \times 10.0 \mu \mathrm{m}$, ornamented with a robust amyloid reticulum, and presence of dendritic pileal terminal elements

Typus: Australia, Victoria, East Gippsland, Rich Forest Management Block, near Jack Road $0.7 \mathrm{~km} \mathrm{~N}$ of junction with Winter Road, Claridge site R19, 29 Feb. 1996, A.W. Claridge AWC201 (holotype MEL 2145804).
Basidiomes hypogeal, sequestrate, 5-15 mm broad, globose to subglobose or irregular, surface dry, smooth to rugulose and irregularly folded radially particularly around the point of attachment to substrate, minutely pruinose and velutinous, pale orange-ochre to almost reddish-brown in folds; pileus in cross-section thin $(\leq 0.5 \mathrm{~mm}$ ), pale orange becoming orangebrown in dried specimens. Hymenophore loculate, locules
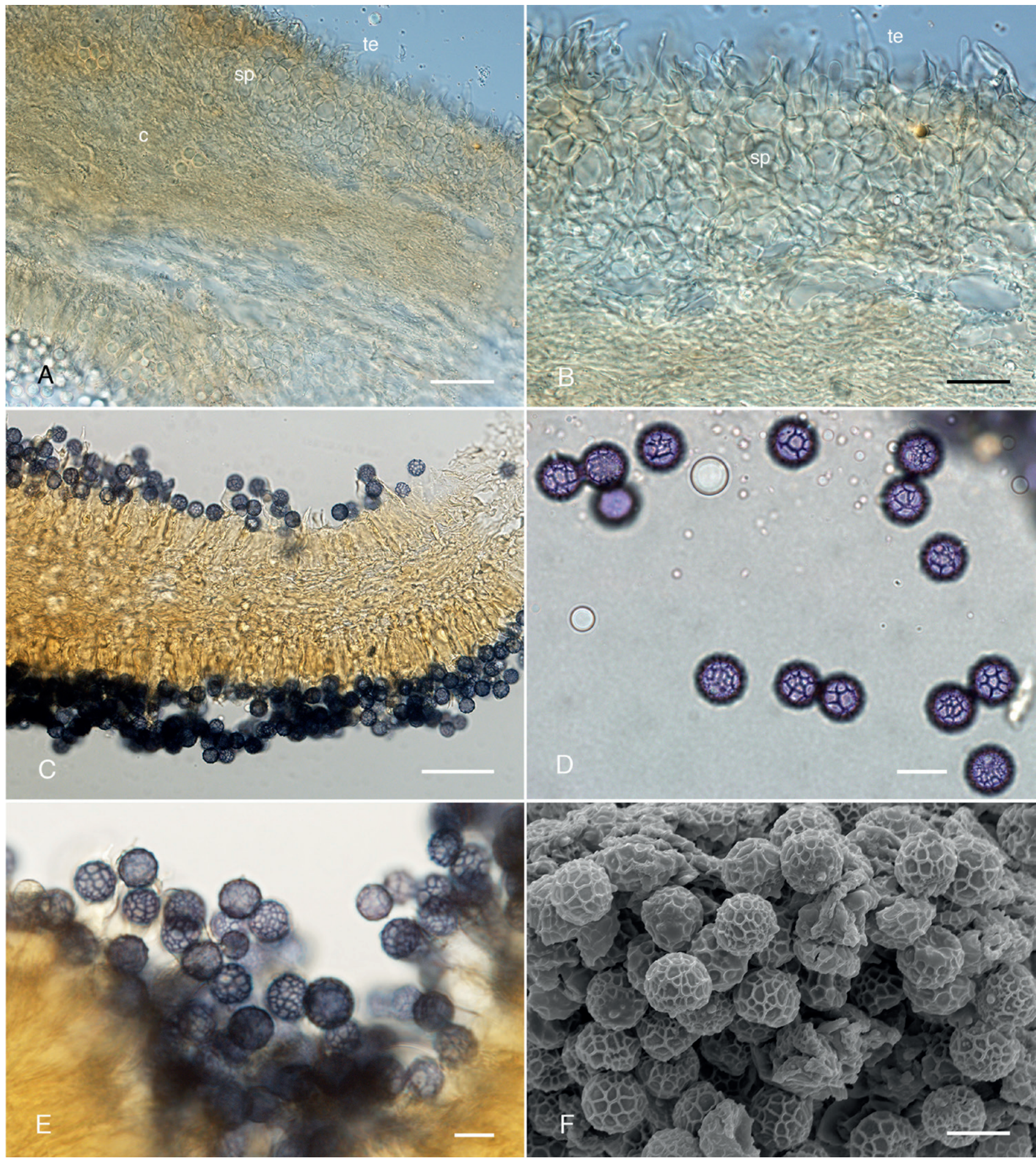

Fig. 5. Lactifluus geoprofluens A. Lampropallisade pileipellis terminal elements (te), subpellis (sp) and context (c). B. Pileipellis terminal elements (te) and subpellis (sp). C. Hymenial trama. D-E. Basidiospores. F. Scanning Electron Microscopy photo micrograph of basidiospores. Scale bars: A, C = 50 $\mu$ m; B = $20 \mu \mathrm{m} ; \mathrm{D}-\mathrm{F}=10 \mu \mathrm{m}$. 
0.5-2 $\mathrm{mm}$, labyrinthine and vaguely radial from columella attachment, pale orange-ochre to reddish orange-brown. Stipe-columella present or absent, much reduced if present and variable, $0.5-3 \mathrm{~mm}$ long $\times 0.5-2 \mathrm{~mm}$ wide, irregularly cylindrical, slightly tapering towards the base, orange-ochre to reddish-brown in patches, minutely pruinose and finely rugulose; context golden to reddish-brown. Latex white, unchanging, abundant along the pileipellis edges and the interior of the hymenophore. Taste mild. Odour not obvious or slightly fishy when fresh. Basidiospores 8.0-10.5 × 8.0-10.0 $\mu \mathrm{m}$ $(\bar{x}=9.85 \pm 0.43 \times 9.79 \pm 0.35, n=40)$, globose to occasionally barely subglobose $[\mathrm{Q}=1.00-1.05(\bar{x}=1.02 \pm 0.02, n=40)$ ], walls amyloid between ridges and plage distally to completely amyloid, ornamentation reticulate with ridge apices to $1 \mu \mathrm{m}$ tall. Basidia 31-52 × 8-14 $\mu \mathrm{m}(\bar{x}=44.18 \pm 5.33 \times 11.25 \pm 1.08$, $n=19), 3-6 \mu \mathrm{m}$ wide at base $(\bar{x}=3.87 \pm 0.54, n=16)$, clavate, mostly 4-spored but occasionally 3-spored; sterigmata 3-10 $\times$ $1-2.5 \mu \mathrm{m},(\bar{x}=7.18 \pm 2.20 \times 2.00 \pm 0.51, n=12)$; basidioles $30-51 \times 6-11 \mu \mathrm{m}(\bar{x}=39.89 \pm 4.46 \times 9.14 \pm 1.41, n=14), 2-5$ $\mu \mathrm{m}$ wide at base $(\bar{x}=3.36 \pm 0.74, n=14)$, cylindrical to barely clavate. Hymenophoral trama comprised of interwoven and parallel tightly packed hyphae $2-3 \mu \mathrm{m}$ diam, interspersed with sinuous laticiferous hyphae $5-7 \mu \mathrm{m} \operatorname{diam}(\bar{x}=5.64 \pm 0.31, n=8)$ and occasional sphaerocytes 30-45 $\times 17-38 \mu \mathrm{m}$; subhymenium composed of chains of 3-4 inflated cells $8-21 \times 6-17 \mu \mathrm{m}(\bar{x}$ $=11.30 \pm 6.70 \times 10.40 \pm 5.72, n=18$ ), laticiferous hyphae present and occasionally extending into pseudocystidia 3-8 $\mu \mathrm{m}$ diam., thin-walled, cylindrical to slightly tortuous, with oily refractive contents. Hymenial cystidia scarce, when present not well differentiated, $31-52 \times 7-11 \mu \mathrm{m}(\bar{x}=46.34 \pm 4.78$ $\times 9.56 \pm 1.16, n=12$ ), subcylindrical tapering to apex, apex mucronate or obtuse, rarely emergent above hymenium, hyaline, thin-walled, contents scattered when present. Pileipellis a lampropalisade; subpellis composed of 3-6 layers of thin-walled polygonal cells 8-19 $\times 7-17(\bar{x}=15.02 \pm 3.28$ $\times 13.20 \pm 2.06, n=12) \mu \mathrm{m}$; lamprocystidia $18-27 \times 2-5 \mu \mathrm{m}$ $(\bar{x}=24.32 \pm 4.12 \times 3.73 \pm 1.02, n=15)$, elongate cylindrical and slightly sinuate, tapering toward apex, apex mucronate or capitate or obtuse, thick-walled, basal width 2-5 $\mu \mathrm{m}(\bar{x}=3.73 \pm$ $1.02, n=15)$, and apical width $1-3 \mu \mathrm{m}(\bar{x}=1.59 \pm 0.35, n=15)$; pileus trama similar to hymenophoral trama, heteromerous, hyphae tightly compressed and interwoven, sphaerocytes not abundant.

Ecology and distribution: Sporulating in February-June in cool temperate forests in NE Victoria. Hypogeal in mixed forests of Eucalyptus maculata, E. muellerana, E. paniculata, E. obliqua, E. cypellocarpa, E. delegatensis, Angophora floribunda, E. longifolia, E. muellerana, E. sieberi, E. baxteri, E. botryoides, with an Acacia cognata, $A$. verticillata, $A$. melanoxylon, $A$. mucronata, A. longifolia, A. mearnsii, A. implexa understorey.

Additional specimens examined: Australia, Victoria, East Gippsland, Mimosa Rocks National Park, Tanja-Tathra Road, $0.5 \mathrm{~km} \mathrm{~N}$ of junction with track Leading to 'Gillards', Claridge Site 40, 7 Jun. 2003, J.M. Trappe, A.W. Claridge, A. Jumpponen AWC5539 (OSC 148637); ibid., Murrangowar Forest Management Block, Bendoc Ridge Road, 0.4 km from Princes Hwy, Claridge Site 66, 2 Jun. 2003, M. Vavrek AWC5306 (OSC 148635); ibid., Cape Conran-Sydenham Inlet Coastal Park, Swampy Creek trail, Crossing of Swampy Creek, Claridge site 128, 31 May 2003, A. Jumpponen AWC5204 (OSC 148634); ibid., East Gippsland, Rich Forest Management Block, junction of Mills Road and Jack Rich Divide Track,
Claridge Retrospective study site R8, 18 Jul. 1996, B. Gunn JMT19613 and AWC452 (OSC 159173); ibid., Nunniong Forest Management Block, off Nunniong Road, Claridge site 106 (relocated 1999), 18 May 2001, T. Lebel AWC3614 (OSC 148633); Ben Boyd National Park, Bittangabee Picnic Area, Bittangabee Creek, Crossing of Walking Track leading N from Picnic Area, Claridge Site 52, 4 Jun. 2003, A. Jumpponen AWC5415 (OSC 148636); Lind National Park, Euchre Valley Road, $0.2 \mathrm{~km} \mathrm{~W}$ of Junction with Lind Park Road, Claridge site 13 (1999 relocation), 29 May 2001, W. Colgan III AWC4223 (OSC 148632).

Notes: This hypogeous, sequestrate fungus is known from cooltemperate mixed species Eucalyptus forest in East Gippsland, Victoria. It differs from the other two sequestrate taxa in often having a distinct columella, radial-locular hymenophore and abundant white latex in all tissues. Lactifluus geoprofluens shares with other species in Lactifluus section Lactifluus the fishy odour, velutinous orange-brown pileus and a lampropalisade pileipellis structure. The basidiospores in Lf. geoprofluens are large (up to $10.5 \times 10 \mu \mathrm{m}$ ) and globose $(\mathrm{Q}=1-1.05)$, unique among the Australian section Lactifluus species.

\section{Section Pseudogymnocarpi}

\section{Lactifluus sp. prov. KV181 Fig. 3F.}

Basidiomes sequestrate, up to $19 \mathrm{~mm}$ broad, globose to subglobose, surface dry, smooth to minutely pruinose and velutinous, pale creamy yellow-tan with some minor slightly darker bruising; pileipellis in section thin $(\leq 0.5 \mathrm{~mm})$, pale tan. Hymenophore loculate, empty locules $0.5-2 \mathrm{~mm}$, labyrinthine, orange-ochre. Stipe absent; columella present, percurrent central strand, $0.5-2 \mathrm{~mm}$ wide, white to pale cream. Latex white turning slightly yellowish, abundant, particularly along the pileipellis edges and the interior of the hymenophore near columella. Taste mild. Odour faint, fungal.

Distribution and habitat: Sporulating in June in cool temperate open woodland in the eastern tablelands of New South Wales. Hypogeal in mixed Eucalyptus forest.

Sequence data: Australia, New South Wales, Gibraltar Range National Park, Mulligans Dr., 16 Jun. 2006, K. Vernes, T. Lebel \& A. O'Malley KV181.

Notes: We provide the image of the basidiome and general notes in the hope that further material will be found. Our analyses show that this taxon is in a strongly supported section Pseudogymnocarpi, however support values within the clade are low (Fig. 2). The majority of agaricoid species currently known within this clade (e.g. Lf. rugatus, Lf. pseudoluteopus, Lf. hygrophoroides) have pale orange to orange-red pilei and stipe, pale coloured lamellae, abundant white latex (Fig. 3E), and an association with Fagaceae or Pinaceae. The lack of microscopic detail, and additional data for Australasian agaricoid taxa or environmental sequences, precludes any statements regarding how well this sequestrate taxon fits in this clade generally.

\section{DISCUSSION}

Both agaricoid and pleurotoid basidiome forms have been found in Lactifluus. In this predominantly agaricoid genus, the 
pleurotoid habit has at least seven different origins in three subgenera: subg. Lactifluus section Gerardii, subg. Lactariopsis and subg. Gymnocarpi (De Crop et al. 2017). No pleurotoid forms are thus far known in Lactarius, but they have been found in Russula (Buyck \& Horak 1999, Henkel et al. 2000). Before the present study, sequestrate forms were not known from Lactifluus, but have evolved many times over in Lactarius; the evolution of a sequestrate basidiome form thus appears to be a rare event in Lactifluus.

All three sequestrate Lactifluus species have a phylogenetic affinity with Southeast Asian taxa. Some, like $L f$. geoprofluens and $L f$. dendriticus, probably evolved in Australia from local ancestors (that have a SE Asian descent - or other way around; cannot be determined at this point). However, the isolated position of $L f$. sp. prov. KV181 is intriguing, as apart from Asian relatives, a European and a North American species are also closely related. This could be due to under sampling of the Australian and Malesian taxa in particular. However, placement within section Pseudogymnocarpi is well supported (BS $98 \%$ ).

While the number of species in Lactarius with sequestrate basidiome forms is much greater (in the order of 25-30 undescribed and nine named species known for Australasia; and a further $22 \mathrm{spp}$. worldwide) than in Lactifluus, all sequestrate taxa also appear to have evolved as independent and isolated incidents within the genus (Trappe et al. 2002, Desjardin 2003, Verbeken et al. 2014a, Sheedy et al. 2016). None of the sequestrate taxa have evolved into a clade containing a large diversity of species with similar basidiome forms, all indicative of relatively recent origin of this basidiome form in Lactarius (Verbeken et al. 2014a) and Lactifluus. The recent description of several sequestrate Lactarius from tropical forests (Verbeken et al. 2014a, b, Buyck et al. 2017), and discovery of hidden diversity of Australasian Lactifluus species lend support to the theory that sequestrate basidiome forms are essentially an excellent way to maintain a fairly constant relative humidity in the enclosed hymenophore, allowing full development of spores to ensue, regardless of arid, seasonally dry or tropical, seasonally waterlogged conditions (Beever \& Lebel 2014).

Unsurprisingly, given the trouble with morphological differentiation of Lactifluus and Lactarius agaricoid species, it is also difficult to distinguish taxa with sequestrate basidiomes. As a broad generalisation, in Australia, the majority of sequestrate Lactarius have yellow to orange to red tinged basidiomes, whereas two of the three Lactifluus taxa described in this study have white to pale cream basidiomes. Pale basidiome colouring is more common in Australasian sequestrate russuloid taxa (Beaton et al. 1984, Lebel \& Trappe 2000, Lebel \& Castellano 2002, Lebel 2002a, b, 2003a, b, Lebel \& Tonkin 2007). General trends in microscopic differentiation between Lactarius and Lactifluus were formulated by Verbeken \& Nuytinck (2013) and can be summarised as: (i) thick-walled elements in the pileipellis and stipitipellis, as well as lamprocystidia, are generally present in Lactifluus and very rarely observed in Lactarius, and (ii) a hymenophoral trama composed of sphaerocytes (as in Russula) is common in Lactifluus but is rarely observed in Lactarius. Both trends don't seem to hold up for the sequestrate Lactifluus species. The loss of thick-walled elements in pileus and hymenium in sequestrate forms could be due to increased folding and loculisation of the hymenophoral trama in combination with loss of ballistospory (i.e. no longer require spacing to allow for spore drop). As the basidiomes are submersed in the soil, dispersal of spores by vectors such as marsupial mammals or insects, is highly likely; by having thin walls the ripe basidiomes would be easier to break open or eat. The highly branched, angular pileal dendritic terminal elements of $L f$. dendriticus are unique. The presence of distinctively shaped pileicystidia or terminal elements is more typical of species of Russula but rarely seen in milkcaps (Verbeken 1996a, Verbeken \& Walleyn 2010). Initially thought to be a contaminant, perhaps a mycenoid mycoparasite or virus, but these terminal elements are found consistently in multiple basidiomes from geographically distant collections, and no DNA sequences for mycenoid taxa were ever recovered as contaminants.

In Lactifluus section Gerardii the majority of species have a brown stipe and pileus contrasting with white mostly distant lamellae, reticulate spore ornamentation $<2 \mu \mathrm{m}$ high, a palisade pileipellis structure, and are generally lacking in macrocystidia (Stubbe et al. 2010, De Crop et al. 2017, 2018). The basidiomes can also be small, white and pleurotoid, with thick-walled terminal elements in the pileipellis (Stubbe et al. 2012, Latha et al. 2016, De Crop et al. 2018). Several of the agaricoid and pleurotoid taxa have latex that changes colour on exposure, however this is not the case for $L f$. dendriticus. While the pale sequestrate basidiomes of $L f$. dendriticus can be easily mistaken for sequestrate russuloid taxa as latex production is variable, the more open form of locules in the hymenophoral tissue tend to suggest a lactarioid taxon. However, it is the unique dendritic pileal terminal elements of $L f$. dendriticus that are intriguing. How does a cystidium evolve from a simple filamentous, perhaps irregularly capitate form, to a highly branched, sharp angled form?

Lactifluus geoprofluens is readily distinguished from Australian and international species in Lf. section Lactifluus by lacking lamprocystidia, having large globose basidiospores and a sequestrate basidiome form. It is the first known sequestrate member of $L f$. section Lactifluus (De Crop et al. 2017). Unfortunately, we do not have an image of the fresh basidiomes of $L f$. geoprofluens, however the field notes all state pale orange-ochre to reddish brown in colour, with slightly paler hymenophoral tissue.

We currently lack data to describe $L f$. sp. prov. KV181 as a new species, but we do provide a picture and a description of field characteristics. It is the first time a non-agaricoid representative of $L f$. subg. Pseudogymnocarpi is documented, and the first Australasian representative in section Pseudogymnocarpi. We look forward to obtaining new material for further examination.

\section{ACKNOWLEDGEMENTS}

We gratefully acknowledge the curation staff at MEL, BRI, and OSC who facilitated access to material for study. The molecular work conducted by James Douch, Luke Vaughan, and Lachlan Tegart was supported by the Jim Willis Studentship program hosted by the Royal Botanic Gardens Victoria, and by some external funding through the 'Fungal Barcode Project' Bioplatforms and the Australian Genome Research Facility. Travel by Jorinde Nuyinck to MEL was supported by Australian Biological Resources grant (RFL217-63). J.A. Cooper is supported by the Strategic Investment Fund of the New Zealand Ministry for Business, Innovation and Employment (MBIE). We also thank J.M. Trappe and A.W. Claridge for providing additional material of $L f$. geoprofluens.

Conflict of interest: The authors declare that there is no conflict of interest. 


\section{REFERENCES}

Beaton G, Pegler DN, Young TWK (1984). Gasteroid basidiomycota of Victoria state, Australia. 2. Russulales. Kew Bulletin 39: 669-698.

Beenken L, Sainge MN, Kocyan A (2016). Lactarius megalopterus, a new angiocarpous species from a tropical rainforest in Central Africa, shows adaptations to endozoochorous spore dispersal. Mycological Progress 15: 58

Beever RE, Lebel T (2014). Truffles of New Zealand: a discussion of bird dispersal characteristics of fruit bodies. Auckland Botanical Society 69: 170-178.

Bougher NL, Lebel T (2001). Sequestrate (truffle-like) fungi of Australia and New Zealand. Australian Systematic Botany 14: 439-484.

Buyck B, Horak E (1999). New taxa of pleurotoid Russulaceae. Mycologia 91: $532-537$

Buyck B, Duhem B, Das K, et al. (2017). Fungal Biodiversity Profiles 2130. Cryptogamie, Mycologie 38: 101-146.

De Crop E, Hampe F, Wisitrassameewong K, et al. (2018). Novel diversity in Lactifluus section Gerardii from Asia: five new species with pleurotoid or small agaricoid basidiocarps. Mycologia 110: 962-984.

De Crop E, Nuytinck J, Van de Putte K, et al. (2017). A multi-gene phylogeny of Lactifluus (Basidiomycota, Russulales) translated into a new infrageneric classification of the genus. Persoonia 38: 58-80.

Desjardin DE (2003). A unique ballistosporic hypogeous sequestrate Lactarius from California. Mycologia 95: 148-155.

Dring DM, Pegler DN (1978). New and noteworthy gasteroid relatives of the Agaricales from tropical Africa. Kew Bulletin 32: 563-569.

Eberhardt U, Verbeken A (2004). Sequestrate Lactarius species from tropical Africa: L. angiocarpus nov. sp. and L. dolichocaulis comb. nov. Mycological Research 108: 1042-1052.

Hall T (2011). Bioedit v7.1.3. http://www.mbio.ncsu.edu/bioedit/ bioedit.html.

Heenan PB, Smissen RD (2013). Revised circumscription of Nothofagus and recognition of the segregate genera Fuscospora, Lophozonia, and Trisyngyne (Nothofagaceae). Phytotaxa 146: 1-31.

Henkel TW, Aime MC, Miller SL (2000). Systematics of pleurotoid Russulaceae from Guyana and Japan, with notes on their ectomycorrhizal status. Mycologia 92: 1119-1132

Katoh K, Rozewicki J, Yamada KD (2019). MAFFT online service: multiple sequence alignment, interactive sequence choice and visualization. Briefings in Bioinformatics 20: 1160-1166.

Kõljalg U, Nilsson RH, Abarenkov K, et al. (2013). Towards a unified paradigm for sequence-based identification of fungi. Molecular Ecology 22: 5271-5277.

Latha KPD, Raj KNA, Farook VA, et al. (2016). Three new species of Russulaceae from India based on morphology and molecular phylogeny. Phytotaxa 246: 61-77.

Lebel T (2002a). The sequestrate Russulales of New Zealand. New Zealand Journal of Botany 40: 489-509.

Lebel T (2002b). Zelleromyces dendriticus a new species from Australia. Australasian Mycologist 20: 4-8.

Lebel T (2003a). Australasian Truffle-like Fungi XV. Cystangium. Australian Systematic Botany 16: 371-400.

Lebel T (2003b). Australasian Truffle-like Fungi XVI. Gymnomyces. Australian Systematic Botany 16: 401-426.

Lebel T, Castellano MA (2002). Type studies of sequestrate Russulales. Part II. Species related to Russula from Australia and New Zealand. Mycologia. 94: 327-354.

Lebel T, Castellano MA, Beever RE (2015). Cryptic diversity in the sequestrate genus Stephanospora (Stephanosporaceae: Agaricales) in Australasia. Fungal Biology 119: 201-228.
Lebel T, Syme A (2012.) Sequestrate species of Agaricus and Macrolepiota from Australia: new species and combinations and their position in a calibrated phylogeny. Mycologia 104: 496-520.

Lebel T, Tonkin JE (2007). Australasian species of Macowanites are sequestrate species of Russula (Russulaceae, Basidiomycota). Australian Systematic Botany 20: 355-381.

Lebel T, Trappe JM (2000). Type studies of sequestrate Russulales. Part I. Generic type species. Mycologia 92: 1188-1205.

Miller MA, Pfeiffer W, Schwartz T (2010). Creating the CIPRES Science Gateway for inference of large phylogenetic trees. In: Proceedings of the Gateway Computing Environments Workshop (GCE), 14 Nov. 2010, New Orleans, LA: 1-8.

Nuytinck J, Verbeken A, Delarue S, Walleyn R (2003). Systematics of European sequestrate lactarioid Russulaceae with spiny spore ornamentation. Belgian Journal of Botany 136: 145-153.

Phookamsak R, Hyde KD, Jeewon R, et al. (2019). Fungal diversity notes 929-1035: taxonomic and phylogenetic contributions on genera and species of fungi. Fungal Diversity 95: 1-273.

Rambaut A (2009). FigTree. http://tree.bio.ed.ac.uk/software/figtree/.

Sang X-Y, Li X-D, Wang Y-W, et al. (2016). Four new sequestrate species of Russulaceae found in China. Phytotaxa 289: 101-117.

Sheedy EM, Martin AF, Ryberg M, et al. (2016). Dating the emergence of truffle-like fungi in Australia, by using an augmented meta-analysis. Australian Systematic Botany 29: 284-302.

Song Y, Zhang JB, Li JW, et al. (2017). Lactifluus sinensis sp. nov. and L. sinensis var. reticulatus var. nov. (Russulaceae) from southern China. Nova Hedwigia. 107: 91-103.

Stamatakis A (2014). RAxML Version 8: A tool for Phylogenetic Analysis and Post-Analysis of Large Phylogenies. Bioinformatics 30: 1312-1313.

Stubbe D, Le HT, Wang XH, et al. (2012). The Australasian species of Lactarius subgenus Gerardii (Russulales). Fungal Diversity 52: 141-167.

Stubbe D, Nuytinck J, Verbeken A (2010). Critical assessment of the Lactarius gerardii species complex (Russulales). Fungal Biology 114: 271-283.

Thiers B (2011) (continuously updated). Index Herbariorum: a global directory of public herbaria and associated staff. New York Botanical Garden's Virtual Herbarium. http://sweetgum.nybg.org/ih/.

Trappe JM, Claridge AW (2003). Australasian sequestrate (trufflelike) fungi 15. New species from tree line in the Australian Alps. Australasian Mycologist 22: 27-38.

Trappe JM, Claridge AW (2008). Arcangeliella claridgei comb. nov. and A. corkii comb. nov. transferred from the genus Zelleromyces. Australasian Mycologist 27: 117.

Trappe JM, Lebel T, Castellano MA (2002). Nomenclatural revisions in the sequestrate russuloid genera. Mycotaxon 81: 195-214.

Verbeken A (1996). Biodiversity of the genus Lactarius Pers. in tropical Africa. Part 1, text. Part 2, plates and maps. Ph.D. dissertation. Biology Department, Ghent University, Belgium.

Verbeken A, Hampe F, Wissitrassameewong K, et al. (2014b). A new angiocarpous Lactarius species from Thailand. Phytotaxa 181: 163170.

Verbeken A, Nuytinck J (2013). Not every milkcap is a Lactarius. Scripta Botanica Belgica 51: 162-168.

Verbeken A, Stubbe D, Van de Putte K, et al. (2014a). Tales of the unexpected: angiocarpous representatives of the Russulaceae in tropical South East Asia. Persoonia 32: 13-24.

Verbeken A, Walleyn R (2010). Fungus Flora of Tropical Africa, volume 2. Monograph of Lactarius in Tropical Africa. Nationale Plantentuin België, Meise.

Vidal JM, Alvarado P, Loizides M, et al. (2019). A phylogenetic and taxonomic revision of sequestrate Russulaceae in Mediterranean and temperate Europe. Persoonia 42: 127-185. 
Wang XH, Stubbe D, Verbeken A (2012). Lactifluus parvigerardii sp. nov., a new link towards the pleurotoid habit in Lactifluus subgen. Gerardii (Russulaceae, Russulales). Cryptogamie, Mycologie 33: 181-190. 